\title{
Effect of Urea on Bovine Serum Albumin in Aqueous and Reverse Micelle Environments Investigated by Small Angle X-Ray Scattering, Fluorescence and Circular Dichroism
}

\author{
Rosangela Itri ${ }^{1}$, Wilker Caetano ${ }^{1}$, Leandro R. S. Barbosa ${ }^{1}$, and Mauricio S. Baptista ${ }^{2}$ \\ ${ }^{1}$ Instituto de Física, Universidade de São Paulo, Cx. Postal 66318, CEP 05315-970, São Paulo, SP, Brazil \\ ${ }^{2}$ Instituto de Química, Universidade de São Paulo, Av. Prof. Lineu Prestes, 748, CEP 05513-970, São Paulo, SP, Brazil
}

Received on 08 October, 2003.

\begin{abstract}
The influence that urea has on the conformation of water-soluble globular protein, bovine serum albumin (BSA), exposed directly to the aqueous solution as compared to the condition where the macromolecule is confined in the Aerosol-OT (AOT - sodium bis-2-ethylhexyl sulfosuccinate)/n-hexane/water reverse micelle (RM) is addressed. Small angle X-ray scattering (SAXS), tryptophan (Trp) fluorescence emission and circular dichroism (CD) spectra of aqueous BSA solution in the absence and in the presence of urea ( $3 \mathrm{M}$ and $5 \mathrm{M})$ confirm the known denaturating effect of urea in proteins. The loss of the globular native structure is observed by the increase in the protein maximum dimension and gyration radius, through the Trp emission increase and maximum red-shift as well as the decrease in $\alpha$-helix content. In RMs, the Trp fluorescence and CD spectra show that BSA is mainly located in its interfacial region independently of the micellar size. Addition of urea in this BSA/RM system also causes changes in the Trp fluorescence (emission decrease and maximum red-shift) and in the BSA CD spectra (decrease in $\alpha$-helix content), which are compatible with the denaturation of the protein and Trp exposition to a more apolar environment in the RM. The fact that urea causes changes in the protein structure when it is located in the interfacial region (evidenced by CD) is interpreted as an indication that the direct interaction of urea with the protein is the major factor to explain its denaturating effect.
\end{abstract}

\section{Introduction}

Water plays important roles in several fields of science and technology including physics, chemistry, biology, medicine and engineering. When life is concerned this importance increases. In fact, it is difficult to find a single phenomenon of biological relevance that is not related somehow with water [1].

The structure of water is still a matter of debate. The most accepted model proposed by Frank and Quist [2] and refined by several authors [3-5] considers it to have two populations: strongly hydrogen bonded or "intact" population, where water molecules are in an ice-like environment, and weakly hydrogen bonded or "broken" population. The energy barrier for the interconversion of these two species was shown to vary with the temperature and this variation could explain several of the "anomalous" properties of water [4].

Biomolecules evolved in aqueous media and therefore their structure are intrinsic related to the water properties [68]. Protein folding, phospholipid self-association, the DNA double helix structure, protein/protein, protein/membranes and protein/DNA interactions are all examples of phenomena intrinsic related to life and that cannot be dissociated from the water structure $[1,6-8]$.

The types of forces responsible for the acquisition of these structures are several including enthalpic ones (electrostatic, hydrogen bonding, Van der Walls, $\pi$-stacking) and entropic ones (hydrophobic interaction). The hydrophobic effect, which is basically driven by an entropy gain, is associated with the decrease in the population of intact water when hydrophobic surfaces self-associate. It is considered to be the major thermodynamic factor related to protein folding and amphiphilic molecules self-association in membranes [7-9].

The way in which these self-assembling processes are affected by several types of electrolytes and non-electrolytes is also a matter of debate [10-12]. The main discussion is whether one specific compound affects the self-assembling by interacting directly with the bioaggregate (direct mechanism) or indirectly by changing the ratio of intact to broken water populations $[5,10,11]$.

There are generally two types of solutes in terms of changing the water structure: structure former agents that are defined as solutes that increase the intact water population and structure breaker agents that decrease the water structure by increasing the broken water population $[12,13]$. The effect of the structure breaker solutes is similar to the effect caused by increasing the temperature of the solution [12,13]. The terms "kosmotrope" (order-maker) and "chaotrope" (disorder-maker) originally denoted solutes that 
stabilized, or destabilized respectively, proteins and membranes. Later, they referred to the apparently correlating property of increasing, or decreasing respectively, the structure of water. Although useful, the terminology may sometimes be misleading as such properties may vary depending either on the solute concentrations or the presence of macromolecules [14]. Also some solutes with less-well defined properties (e.g. urea) are sometimes classified as kosmotropes [14] and sometimes as chaotropes [15].

Ionic kosmotropes and chaotropes should be treated differently from non-ionic solutes. Generally ionic behavior parallels Hofmeister series. Large singly charged ions, with low charge density (e. g. $\mathrm{H}_{2} \mathrm{PO}_{4}^{-}, \mathrm{HSO}_{4}^{-}, \mathrm{I}^{-}, \mathrm{Cl}^{-}$, $\mathrm{NO}_{3}^{-}, \mathrm{NH}_{4}^{+}, \mathrm{Cs}^{+}, \mathrm{K}^{+}$), which exhibits weaker interactions with water than water with itself, are chaotropes, whereas small or multiply-charged ions, with high charge density, are kosmotropes (e.g. $\mathrm{SO}_{4}^{2-}, \mathrm{HPO}_{4}^{2-}, \mathrm{Mg}^{2+}, \mathrm{Ca}^{2+}, \mathrm{Li}^{+}$, $\mathrm{Na}^{+}, \mathrm{OH}^{-}$), which exhibits stronger interactions with water molecules than water with itself. An extensive review of the Hofmeister series has been presented by Collins and Washabaugh [16].

Non-ionic kosmotropes are very soluble well-hydrated molecules, having no net charge and enforcing extensive hydrogen bonding, that stabilize the structure of macromolecules in solution. Trehalose, glucose, glycine betaine, t-butanol, proline, are all examples of kosmotrope agents. Conversely, chaotropes increase the population of broken water decreasing the hydrogen-bonded network of water. It allows macromolecules to have more structural freedom and facilitates protein denaturation.

In particular, the effect of urea on the structure of biopolymer and bioaggregate is not well understood in the molecular level despite the expressive number of works published in this area. Urea is the most widely used denaturating agent, being extensively applied in biochemistry, not only to denaturate proteins at high urea concentration, but also to promote controlled folding. Whether urea interacts directly in the biopolymer promoting a better solvation of the apolar residues or indirectly by decreasing the water structure is the main topic of discussion in this research area [10,11].Other mechanisms have also been proposed to explain the effect of urea in biopolymers and bioaggregates $[17,18]$.

In order to understand the effect of urea on the molecular level several biomimetic systems are used. Usually they are relatively easy to prepare and understand. The effect of urea in several biomimetic systems (vesicles, micelles and reverse micelles) has provided important contributions to this field. Reverse micelles (RMs) are spontaneously formed colloidal systems with a surfactant monolayer separating a water pool from a bulk organic phase. In general, the radius of the encapsulated water pool is nearly proportional to the molar ratio of water to surfactant denoted as W [19]. It has been demonstrated, by means of infrared and NMR techniques, the existence of different water species in RMs coexisting and exchanging rapidly [19-22]. They are related to counterion and headgroup-bound water that lack the normal hydrogen-bonded structure and bulk-like water residing in the core of the aqueous-pool [22]. Addition of water to the system, beyond the hydration capability of counterions and headgroups, increases the content of the bulk-like water and, therefore, the properties of the water cavity resemble those of bulk aqueous media [20]. Because AOT RMs offer controlled water environments in apolar media, they have been used to mimic the water-biomembrane interface and to investigate membrane interaction with urea, short biologically active peptides, proteins and enzymes [17,23-26].

Perhaps the most studied model of globular protein is Serum albumin. It is synthesized by the liver in mammals with half-life in the circulatory system of $c a$. 19 days. It corresponds to the most abundant protein, accounting for 60 $\%$ of the total globular protein in blood plasma [27-30]. Its function is associated to the binding and transport of several small molecules such as fatty acids, dyes, metals, amino acids, as well as several pharmaceutical compounds [2731]. Its primary structure is well known and it is constituted by around 585 amino acid residues, whereas its secondary structure is constituted by $67 \%$ alpha helix and 17 disulfide bridges, that confer to the protein a relatively strong stability [27,29].

Therefore, with a view of investigating the effect of urea in biopolymers and bioaggregates, the BSA secundary structure was investigated in aqueous solution upon addition of high urea concentration ( 3 and $5 \mathrm{M}$ ), by spectroscopic fluorescence measurements and circular dichroism (CD). Small angle X-Ray scattering (SAXS) was also performed to reveal modifications on the whole protein conformation. Further, all these results are compared with those obtained from protein-containing reverse micelles composed of RM with $\mathrm{W}=10$, which is a condition where strong interaction between water molecules and surfactant polar heads is expected [22,32,33], and practically no bulk-like water is present.

\section{Material and Methods}

\subsection{Materials}

Aerosol-OT (AOT, sodium bis (2-ethylhexyl) sulfosuccinate) of $99 \%$ purity was obtained from Sigma-Aldrich Chemical Co. and used as received. Anhydrous hexane HPLC grade (Merck), the monobasic sodium phosphate salt (Fischer Chemical Co), urea (Carlo Erba) and Bovine Serum Albumin protein (BSA - 66.3 kDa -from Acros Organics Co.) were used as purchased.

Samples. All BSA-containing aqueous solutions in the absence and presence of 3 and $5 \mathrm{M}$ urea were prepared in $10 \mathrm{mM}$ phosphate buffer at $\mathrm{pH} 7.2$ (above its $\mathrm{pI}=4.9$ ). The samples were made just before the measurements and the BSA concentration was $150 \mu \mathrm{M}(10 \mathrm{mg} / \mathrm{ml})$ for SAXS, and $11 \mu \mathrm{M}$ for fluorescence and CD spectra (circa $5 \mu \mathrm{M}$ in the $\mathrm{RM})$. The reverse micellar solutions were prepared by injecting a desirable volume of phosphate buffer $(10 \mathrm{mM}, \mathrm{pH}$ 7.2, also in the presence and absence of 3 and $5 \mathrm{M}$ urea) into a $100 \mathrm{mM}$ AOT/hexane solution. For BSA-containing 
micellar solutions, a buffered stock solution with protein in the concentration range of $10 \mathrm{mg} / \mathrm{ml}$ was injected. All the injected volume was considered as water (or water/urea) and used to calculate W ([water]:[AOT]) value of 10. It should be remarked that this approximation is valid only if the protein concentration is low and/or the protein volume is negligible compared to the water volume.

\subsection{Methods}

\section{Steady-state Fluorescence and Circular Dichroism}

Fluorescence spectra were obtained in a Spex Fluorolog Spectrofluorometer model DM3000F equipped with four adjustable slits and a 450W Xenon lamp, with excitation wavelength at $295 \mathrm{~nm}$, with optical density of 0.07 to avoid inner filter effect, and a $1 \mathrm{~cm}$ pathway cuvette. The observed fluorescence in proteins is, basically, given by the tryptophan residues emission. Bovine albumin has two tryptophan residues, $\mathrm{W}^{131}$ and $\mathrm{W}^{214}$, probably located in hydrophobic domains. It has been already documented that the $\mathrm{W}^{214}$ residue in the human serum albumin is located in a hydrophobic pocket in subdomain IIA, while the second one in the BSA is buried in a hydrophobic pocket and it has been proposed to lie near the surface [34]. Changes in the tryptophan's microenviroment can, therefore, be accompanied by the fluorescence emission band that occurs in the range of 320-360 $\mathrm{nm}$.

The CD spectra were measured in a JASCO-720 spectropolarimeter. The samples were contained in quartz cuvettes with path length of 0.2 and $0.5 \mathrm{~mm}$. The spectra have been smoothed and are presented in units of mean residue molar ellipticity. All measurements were performed at $24 \pm$ $1{ }^{\circ} \mathrm{C}$.

\section{Small Angle X-Ray Scattering (SAXS)}

The experiments were performed at National Synchrotron Light Laboratory (LNLS), Campinas, Brazil, using a detector-to-sample distance of $930 \mathrm{~mm}$. The scattering vector amplitude $q$ is defined as $q=4 \pi \sin \theta / \lambda$, being $2 \theta$ the scattering angle, and $\lambda$ the $\mathrm{x}$-ray wavelenght of $1.608 \AA$. The experimental intensities were corrected for background, buffer contributions, sample's attenuation and detector homogeneity.

The scattering intensity from a set of monodisperse particles randomly distributed, is given by [35-37]

$$
I(q)=\gamma n_{p}(\Delta \rho)^{2} V^{2} P(q) S(q)
$$

where $\gamma$ is a factor related to the instrumental effects; $\mathbf{n}_{p}$ corresponds to the particle number density; $\Delta \rho$ is the electron density contrast between the scattering particle and the medium and $V$ is the scattering particle volume; $P(q)$ is the normalized particle form factor $(P(0)=1)$ and $S(q)$ is the interparticle interference function.

In the absence of interference effects, a Fourier transform connects $P(q)$, and hence $I(q)$, to the pair distance distribution function, $p(r)$, the probability of finding a pair of small elements at a distance $r$ within the entire volume of the scattering particle as $[35,37]$

$$
p(r)=\left(\frac{1}{2 \pi^{2}}\right) \int_{0}^{\infty} I(q) q r \sin (q r) d q
$$

This function provides information about the shape of the scattering particle as well as its maximum dimension, $D_{\max }$, accounted for at certain $r$ value where $p(r)$ goes to zero. Moreover, the particle radius of gyration $R g$ value is given by [37]

$$
R g^{2}=\frac{\int_{0}^{D \max } p(r) r^{2} d r}{2 \int_{0}^{D \max } p(r) d r}
$$

In this work, we make use of the GNOM program [38] to calculate $p(r)$ from the SAXS curves.

\section{Results and Discussion}

Figure 1 shows the SAXS curves of BSA in aqueous solution in the absence and presence of 3 and $5 \mathrm{M}$ urea. As one can see, urea induces changes in the protein conformation in such a way that the scattering profiles are distinct and displaced towards low $q$ values, characteristics of some protein unfolding. This finding is corroborated by $p(r)$ function analysis (Fig. 2) that shows that the protein maximum dimension $D_{\max }$ increases from circa $80 \AA$ (corresponding to folded-native BSA [39]) to circa $135 \AA$ and $240 \AA$ when $3 \mathrm{M}$ and $5 \mathrm{M}$ urea is added to the system, respectively. Concurrently, the BSA radius of gyration $R g$ changes from $30 \AA$ (native BSA [39]) to $45 \AA$ and $72 \AA$ in the absence and presence of 3 and $5 \mathrm{M}$ urea, respectively. Under the influence of $3 \mathrm{M}$ urea it is clear that the protein presents some expansion in aqueous solution, however it must preserve most of its structure because the greater frequency of distances in $p(r)$ (at $r \cong 40 \AA$ ) remains unaltered. On the other hand, BSA unfolds to a larger extent at concentration of $5 \mathrm{M}$ urea, as clearly observed by the displacement of greater frequency of $\mathrm{p}(\mathrm{r}$ ) distances towards larger $\mathrm{r}$ values (Fig. 2).

The conformational changes in BSA induced by urea, as explored by fluorescence and CD spectroscopy, are shown in Figs. 3 and 4, respectively. The fluorescence maximum emission of tryptophan (Trp) is sensitive to microenvironment polarity and specific solvent interactions [40,41]. As it is observed in Fig. 3A, the maximum wavelength $\left(\lambda_{\max }\right)$ for BSA tryptophan fluorescence was $327.6 \mathrm{~nm}$ in phosphate buffer. When the protein is in the presence of $3 \mathrm{M}$ and $5 \mathrm{M}$ urea-containing aqueous solution (Fig. 3A), $\lambda_{\max }$ is shifted to $332.6 \mathrm{~nm}$ (red-shift). These shifts indicate that the tryptophan residues of the BSA are exposed to a more polar environment. 


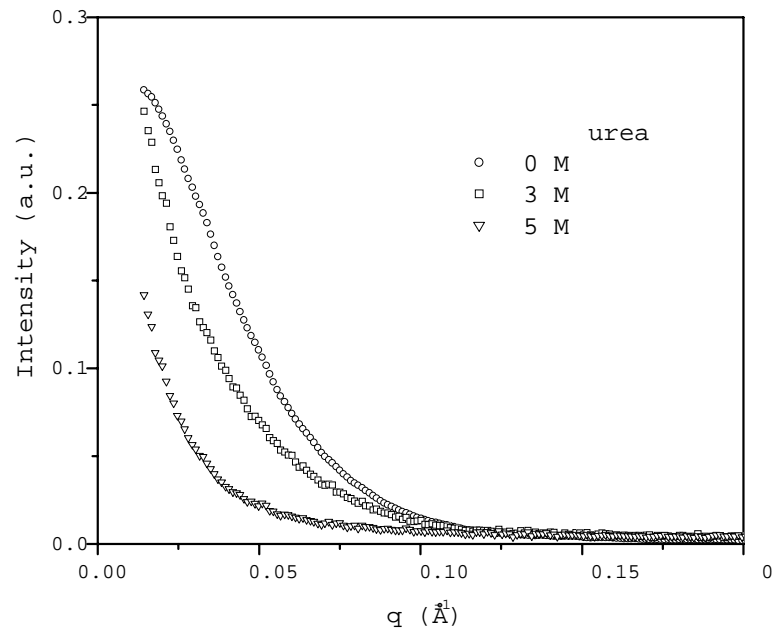

Figure 1. Experimental small angle X-ray scattering curves obtained at $24{ }^{\circ} \mathrm{C}$ in $10 \mathrm{mM}$ phosphate buffer at $\mathrm{pH} 7.2$ for BSAcontaining aqueous solution in the absence and presence of 3 and $5 \mathrm{M}$ urea.

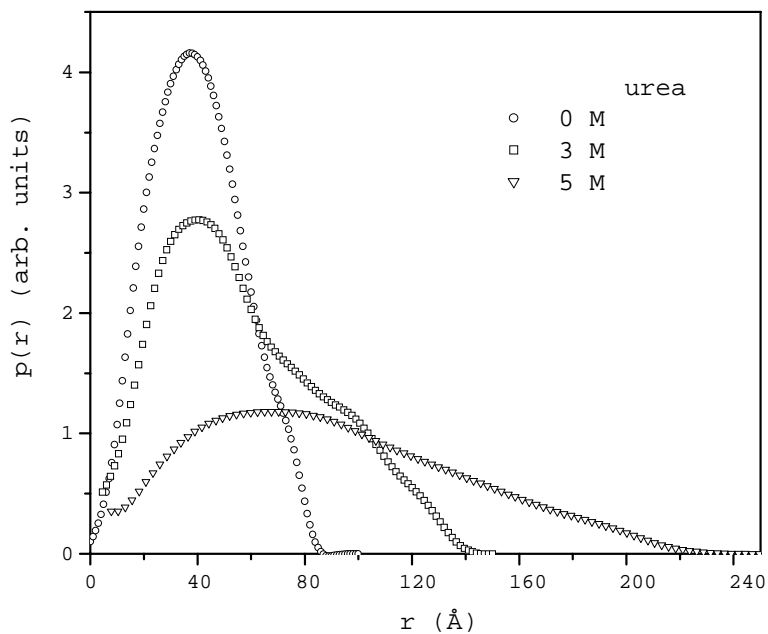

Figure 2. $p(r)$ functions obtained from the SAXS curves of samples composed of BSA in $10 \mathrm{mM}$ phosphate buffer solution in the absence and presence of 3 and $5 \mathrm{M}$ urea, at $\mathrm{pH} 7.2,24{ }^{\circ} \mathrm{C}$.

The increase in fluorescence emission in the presence of urea is also compatible with this hypothesis. In folded proteins the tryptophan emission is usually suppressed by the presence of charged residues in close proximity, which is absent in the unfolded protein [40]. Note that the addition of urea to a BSA solution causes a large increase in the Trp emission (Fig. 3A).

The CD spectrum for BSA in aqueous solution is characteristic of macromolecules with high $\alpha$-helical content, monitored by the two well-defined ellipticities values at 208 and $222 \mathrm{~nm}$ [42]. As one can see from Fig. 4, the helical structure diminishes from $66 \%$ to circa $64 \%$ and $33 \%$, respectively in $3 \mathrm{M}$ and $5 \mathrm{M}$ urea-aqueous solution, in good agreement with literature [24].
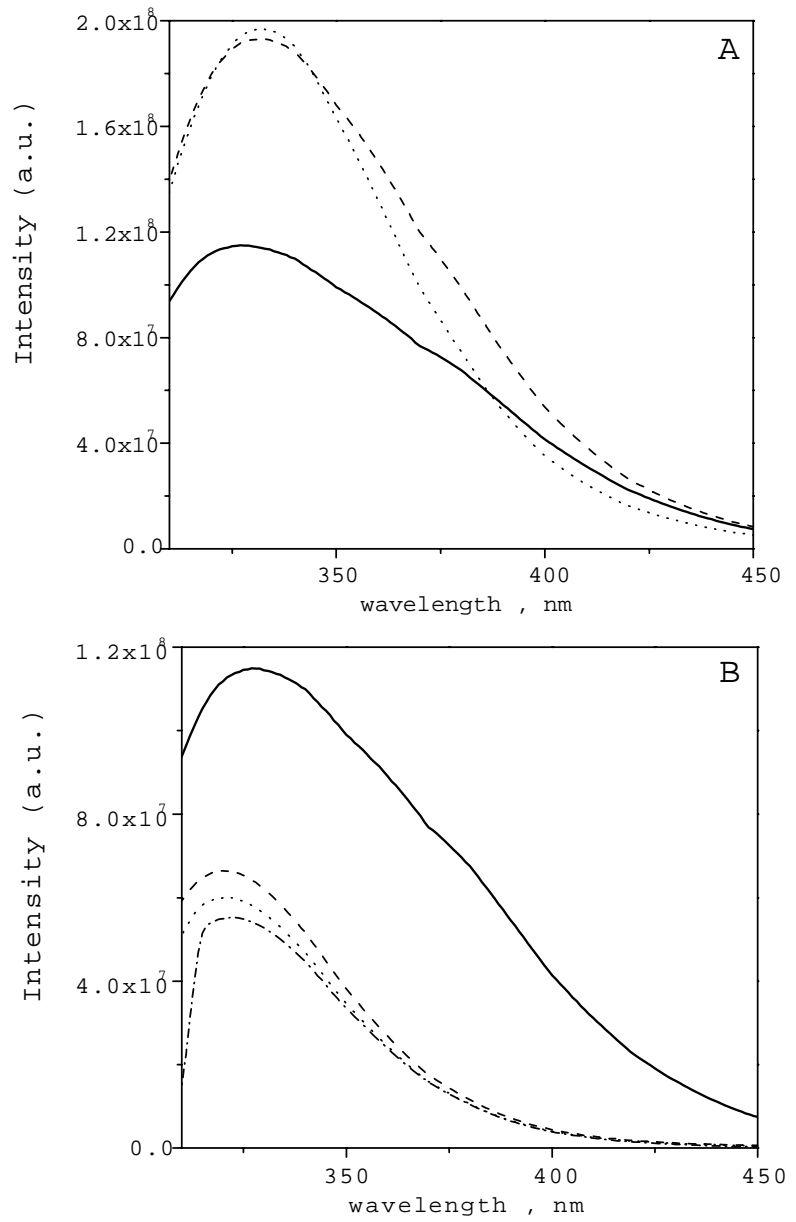

Figure 3. Fluorescence spectra of BSA $\left(1.1 \times 10^{-5} \mathrm{M}\right)$ in $10 \mathrm{mM}$ phosphate buffer, pH 7.2 (solid line), urea-aqueous $3 \mathrm{M}$ solution (dashed-line) and 5M (dotted line). (B) Fluorescence spectra of BSA $\left(1.1 \times 10^{-5} \mathrm{M}\right)$ in $10 \mathrm{mM}$ phosphate buffer, $\mathrm{pH} 7.2$ (solid line); of $[\mathrm{BSA}]=0.5 \times 10^{-5} \mathrm{M}$ in pure AOT/ $\mathrm{n}$-hexane reverse micelles at $\mathrm{W}=10$ (dashed line); in RM with $3 \mathrm{M}$ urea (dotted line) and RM with $5 \mathrm{M}$ urea (dashed-dotted line). Excitation at $\lambda=295 \mathrm{~nm}$.

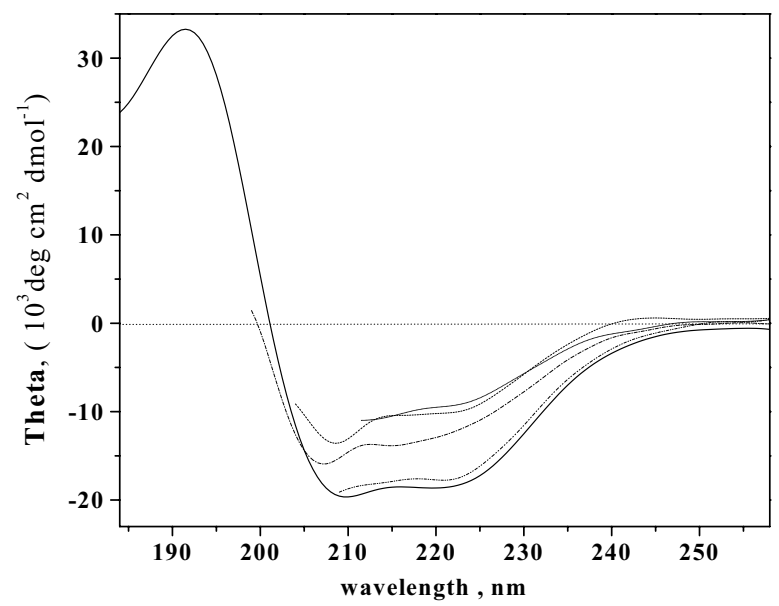

Figure 4. CD spectra of BSA $\left(0.5 \times 10^{-5} \mathrm{M}\right)$ in $10 \mathrm{mM}$ phosphate buffer, pH 7.2 (solid line); in urea-aqueous $3 \mathrm{M}$ solution (dasheddotted-dotted line), in AOT/ n-hexane reverse micelles at $\mathrm{W}=10$ (dashed-dotted line) and containing urea 3M (short-dashed line); 
in urea-aqueous 5M solution (short-dotted line).

Therefore, the combined results give support to conclude that although the presence of $3 \mathrm{M}$ urea induces some protein unfolding, as revealed by SAXS, its secondary structure remains almost unaltered in terms of helical structure as evidenced by $\mathrm{CD}$. On the other hand, $5 \mathrm{M}$ urea has a pronounced effect on protein structure: the $\alpha$-helical content suffers a remarkable decreasing accompanied by a significant protein conformational change as revealed by the values observed for macromolecule radius of gyration $(R g=$ $72 \AA)$ and maximum dimension $\left(D_{\max }=240 \AA\right)$ as well as from $p(r)$ function behavior (Fig. 2B).

The protein unfolding caused by urea in an aqueous environment may be due to the direct or indirect mechanisms or even both. Therefore, it is interesting to look for systems in which one of these mechanisms is not present. If the protein in study is included in an interfacial region, the effect of urea in the structure of water (indirect mechanism) may not be important for the protein structure as far as the interfacial region is not affected itself. RMs allows a microenvironment where this possibility can be tested. The effect of urea in RMs has already been studied. Urea affects the interparticle interactions, but the structure of the independent micelles is kept unchanged [32]. It should also be remarked that the BSA incorporation does not affect the micelle morphology (size and shape) in $\mathrm{W}=10$ as observed by SAXS (data not shown).

The fluorescence behavior of Trp amino acid analogue 7-azatryptophan (7ATrp) has been studied in AOT RM in nheptane [41]. Upon increasing $\mathrm{W}$ from 0.5 to 50 , it has been demonstrated that the emission maximum wavelength undergoes a pronounced red shift (from $\lambda_{\max } \sim 370$ up to $390 \mathrm{~nm}$ ). The effect has been explained [41] by considering a displacement of the 7ATrp molecules solubilized near the inner periphery of the micellar core towards the polar water pools RM as the micellar size increases. In contrast, in the case of BSA, it has been observed that the wavelength of maximum emission of Trp does not change as a function of $\mathrm{W}$ (data not shown), suggesting that the protein is strongly attached in a micellar interface independently of the size of the AOT RM.

The influence of urea on the spectroscopic properties of protein entrapped in RMs is shown in Fig. 3B and in Fig. 4. Fig. 3B shows the fluorescence emission spectrum for BSA in aqueous solution in comparison to those obtained in the presence of AOT/n-hexane/water reverse micelles in the absence and presence of 3 and $5 \mathrm{M}$ urea. In contrast to the effect observed in water, $\lambda_{\max }$ decreases from $327.6 \mathrm{~nm}$ for protein in aqueous solution to $320.2 \mathrm{~nm}$ (blue-shift) for BSA confined in RMs. In this case, BSA Trp residues probe a more apolar environment in the RM, probably due to the protein association with the micellar interfacial region $[23,28,40]$. It is interesting to note that in the presence of $3 \mathrm{M}$ urea in AOT reversed micelles, there is a small red shift in the wavelength of maximum emission of $\operatorname{Trp}\left(\lambda_{\max }=320.5 \mathrm{~nm}\right)$. This red-shift is increased in the presence of $5 \mathrm{M}$ urea $\left(\lambda_{\max }=322.0 \mathrm{~nm}\right)$ in comparison with the $\lambda_{\max }$ observed for BSA in AOT RM without urea $\left(\lambda_{\max }=320.2 \mathrm{~nm}\right)$. The fact that urea affects the emission of Trp when BSA is located in the interfacial region, suggests that urea tends to interact directly with the protein. In other words the change in environment sensed by the Trp cannot be due to the indirect exchange in the water structure due to its interaction with water.

Analysis of the intensity of Trp emission is also compatible with this hypothesis. The decrease in the Trp emission upon incorporation of BSA in the RM (Fig. 3B) is just a concentration effect. The BSA concentration is smaller in the RM solution. However the addition of $3 \mathrm{M}$ and $5 \mathrm{M}$ urea by keeping the BSA concentration constant, slightly decreases the Trp emission, suggesting that urea unfolds the protein in the interface (Fig. 4). This effect is the opposite to that observed in water, where unfolding increases the Trp emission. This can be understood considering that the interface has a large concentration of charged groups that can suppress the Trp emission. Results from CD (Fig. 4) exhibit a decreasing of helical structure from $66 \%$ to $48 \%$ upon the incorporation of BSA into the AOT RM without urea, in good agreement with literature [24]. Such finding can be also correlated to surfactant binding that induces some protein unfolding [39]. The mechanism of protein unfolding by the interface is likely to be the promotion of a better solvation of apolar residues. Still, the presence of $3 \mathrm{M}$ urea in the RMs water pool induces a further decrease in the BSA helical structure to circa $36 \%$, also indicating the direct interaction of urea with BSA in the interfacial region. This decrease in secondary structure is quite larger than that observed in pure aqueous urea solution, in which BSA conserves $64 \%$ helical structure at $3 \mathrm{M}$ urea

\section{Conclusion}

The denaturation of the BSA native structure in aqueous solution by urea, was studied by SAXS, Trp fluorescence and CD. The comparative analysis of structural (SAXS) and spectroscopic data, provided information on the magnitude of BSA denaturation in 3 and $5 \mathrm{M}$ urea concentrations. When BSA is included in the interface of AOT RM, the spectroscopic data (Trp emission maxima and intensity as well as $\mathrm{CD}$ ), show that it is unfolded by the presence of 3 and $5 \mathrm{M}$ urea. The decrease in the BSA secondary structure caused by urea is more intense in RM compared with the effect observed in aqueous solution. Considering that the indirect mechanism of protein unfolding cannot be important in this condition, we have provided clear evidence that the direct interaction of urea with BSA is the major contributor to the protein unfolding caused by this agent.

\section{Acknowledgements}

The authors thank to National Laboratory of Synchrotron Light (LNLS, Campinas, Brazil) for the use of their facilities and to Dra. Shirley Schreier and C. Shida, from the 
Institute of Chemistry (USP), for the use of the CD equipment. W. Caetano and L.R.S. Barbosa are recipients of a pos-doc grant from FAPESP and IC/PIBIC from CNPq, respectively. Research fellowships to M. S. Baptista and R. Itri from $\mathrm{CNPq}$ are also acknowledged.

\section{References}

[1] M. W. Denny, Air and Water: The Biology and Physics of Life's Media, Princeton University, Princeton 1993.

[2] H. S. Frank, J. Quist, J. Chem. Phys. 34, 604 (1961).

[3] G. E. Walrafen, M. R. Fisher, M. S. Hokmabadi, and W. H. Yang, J. Chem. Phys. 85, 6970 (1986).

[4] (a) M. P. Bassez, J. Lee, and G. W. Robinson, J. Phys. Chem. 91, 5818 (1987). (b) G. W. Robinson, J. Lee, and M. P. Bassez, J. Chem. Soc., Faraday Trans. 2 82, 2351 (1986). (c) G. W. Robinson, J. Lee, and M. P. Bassez, Chem. Phys. Lett. 137, 376 (1987).

[5] M. S. Baptista, C. D. Tran, J. Phys. Chem. 99, 12952 (1995).

[6] C. de Duve, Am. Sci. 83, 428 (1995).

[7] F. H. Stillinger, Science 209, 451 (1980).

[8] J. Ladburry, Chem. Biol. 3, 451 (1996).

[9] E. Frieden, J. Chem. Educ. 52, 754 (1975).

[10] O. Enea, C. Jollcoeur, J. Phys. Chem. 86, 3870 (1982).

[11] E. Liepinsh, G. Otting, J. Am. Chem. Soc. 116, 9670 (1994).

[12] J. Stangret, J. Kostrowicki, J. Sol. Chem. 17, 165 (1988).

[13] R. Bhanumathi, S. K. Vijayalakshamma, J. Phys. Chem. 90, 4666 (1986).

[14] P. M. Wiggins, Cell. Mol. Biol. 47, 735 (2001).

[15] J. R. De Xammar Oro, J. Biol. Phys. 27, 73 (2001).

[16] K. D. Collins, M. W. Washabaugh, Q. Rev. Biophys. 18, 324 (1985).

[17] G. D. Dias, F. H. Florenzano, W. F. Reed, M. S. Baptista, S. M. B. Souza, E. B. Alvarez, H. Chaimovich, I. M. Cuccovia, C. L. C. Amaral, C. R. Brasil, L. S. Romsted, and M. J. Politi, Langmuir 18, 319 (2002).

[18] J. Gradolnik, Y. Maréchal, J. Mol. Struct. 615, 177 (2002).

[19] Q. Li, T. Li, J. Wu, and N. F. Zhou, J. Colloid Interface Sci. 229, 298 (2000).

[20] Q. Li, T. Li, and J. Wu, J. Phys. Chem. 104, 9011 (2000).

[21] T. K. Jain, M. Varshney, and A. Maitra, J. Phys. Chem. 93, 7409 (1989).
[22] D. Fioretto, M. Freda, S. Mannaioli, G. Onori, and A. Santucci, J. Phys. Chem. 103, 2631 (1999).

[23] D. M. Davis, D. McLoskey, D. J. S. Birch, P. R. Gellert, R. S. Kittlety, and R. M. Swart, Biophys. Chem. 60, 63 (1996).

[24] K. Takeda, K. Harada, K. Yamaguchi, and Y. Moriyama, J. Colloid Interface Sci. 164, 382 (1994).

[25] G. X. Cheng, F. Shen, L. F. Yang, L. R. Ma, Y. Tang, K. D. Yao, and P. C. Sun, Mater. Chem. Phys. 56, 97 (1998).

[26] E. P. Melo, P. Fojan, J. M. S. Cabral, and S. B. Petersen, Chem. Phys. Lipids 106, 181 (2000).

[27] D. Carter, B. Chang, J. X. Ho, K. Keeling, and Z. Krishnasami, Eur. J. Biochem 226, 1049 (1994).

[28] J. R. Brown, P. Shockley, Lipid-Protein Interactions, vol. 1, Wiley, New York, 1982.

[29] T. Peters, All about albumins: biochemistry, genetics and medical applications. San Diego, 1996.

[30] X. Min He, D. Carter, Nature 358, 209 (1992).

[31] S. Curry, H. Mandelkow, P. Brick, and N. Franks, Nat. Struct. Biol. 5, 827 (1998)

[32] C. L. C. Amaral, R. Itri, and M. J. Politi, Langmuir 12, 4638 (1996).

[33] R. Itri, C. L. C. Amaral, and M. J. Politi, J. Chem. Phys. 111, 7668 (1999).

[34] E. L. Gelamo, M.Tabak, Spectrochimica Acta 56, 2255 (2000).

[35] A. Guinier, G. Fournet, Small angle scattering of X-rays, Wiley, New York, 1955.

[36] M. Kotlarckyk, S. H. Chen, J. Chem. Phys. 79, 2461 (1983).

[37] O. Glatter, O. Kratky, Small Angle X-ray Scattering, Academic Press, London, 1982.

[38] A. V. Semenyuk, D. I. Svergun, J. Appl. Cryst. 24, 537 (1991).

[39] S. F. Santos, D. Zanette, and R. Itri, J. Colloid Interface Sci. 262, 400 (2003).

[40] S. S. Lee, D. J. Kiserow, and L. B. McGown, J. Colloid Interface Sci. 193, 32 (1997).

[41] P. K. Sengupta, J. Guharay, Biochem. and Biophys Res. Commun. 219, 388 (1996).

[42] S. M. Kelly, N. C. Price, Biochim. et Biophys. Acta 161, 1338 (1997). 